\title{
Prevalência de distúrbios osteomusculares e fatores associados na população idosa:
} revisão sistemática

\section{Prevalence of musculoskeletal disorders and associated factors in the elderly population: a sistematic review}

\author{
(DBuna Duarte Nunes de Oliveira ${ }^{1}$, (D)Aliteia Santiago Dilélio ${ }^{1}$, (DMirelle de Oliveira Saes²
}

1 Universidade Federal de Pelotas - UFPEL

2 Universidade Federal do Rio Grande - FURG

\section{Correspondência}

Bruna Duarte Nunes de Oliveira

E-mail: bduartenunes@gmail.com

Submetido: 06 Março 2020 Aceito: 19 Novembro 2020

\section{Como citar}

Oliveira BDN, Dilélio AS, Saes MO. Prevalência de distúrbios osteomusculares e fatores associados na população idosa: revisão sistemática. Acta Fisiatr. 2020;27(4):248-255.

DOI: $10.11606 /$ issn.2317-0190.v27i4a167452

\begin{abstract}
RESUMO
Objetivo: Descrever qual a prevalência de distúrbios osteomusculares e fatores associados na população idosa a partir de uma revisão sistemática. O envelhecimento da população é um fenômeno mundial e acarreta em um aumento progressivo da prevalência de idosos e de doenças crônico degenerativas. Método: Para a seleção dos artigos, efetuou-se busca nas bases de dados PUBMED, SciELO, LILACS e WEB OF SCIENCE durante o mês de agosto de 2018. Foram utilizados os descritores indexados nos Descritores em Ciências da Saúde (DeCS) e no Medical Subject Headings (MeSHTerms). Resultados: Inicialmente identificou-se 6.290 artigos, excluindo-se 19 duplicatas. Realizou-se a leitura dos títulos no Software Mendeley, selecionado-se 132 artigos para a leitura dos resumos e, após 60 artigos para a leitura na íntegra. Ao final foram selecionados 21 artigos, excluindo-se aqueles que não respondiam à questão norteadora e não tinham um desfecho equivalente à proposta dessa revisão. Evidências demonstraram que há uma grande variabilidade na prevalência dos distúrbios osteomusculares em idosos, variando de $0,6 \%$ a $72,1 \%$ e, nos instrumentos utilizados para aferi-los. Apesar da literatura consolidar a associação dos distúrbios osteomusculares com sexo feminino e idade avançada, não há consenso acerca da sua associação com a capacidade funcional e a utilização de serviços de saúde. Conclusão: Observou-se ainda que o alcoolismo, o tabagismo, a inatividade física, a obesidade e a hipertensão arterial sistêmica apresentam associação com os distúrbios osteomusculares em idosos. As elevadas prevalências identificadas remetem a importância do planejamento de ações em saúde que visem preparar os profissionais e os serviços públicos de saúde para atender esta parcela crescente da população.
\end{abstract}

Palavras-chave: Idoso, Doenças Musculoesqueléticas, Prevalência

\begin{abstract}
Objective: To describe the prevalence of musculoskeletal disorders and associated factors in the elderly population from a systematic review. Population aging is a worldwide phenomenon and leads to a progressive increase in the prevalence of the elderly and chronic degenerative diseases. Methods: For the selection of articles, a search was carried out in the PUBMED, SCIELO, LILACS and WEB OF SCIENCE databases during August 2018. The descriptors indexed in the Health Sciences Descriptors (DeCS) and in the Medical Subject Headings were used (MeSHTerms). Results: Initially, 6,290 articles were identified, excluding 19 duplicates. The titles were read in the Mendeley Software, 132 articles were selected for reading the abstracts and, after 60 articles for reading in full. At the end, 21 articles were selected, excluding those that did not answer the guiding question and did not have an outcome equivalent to the proposal of this review. Evidence has shown that there is great variability in the prevalence of musculoskeletal disorders in the elderly, ranging from $0.6 \%$ to $72.1 \%$ and in the instruments used to measure them. Despite the literature consolidating the association of musculoskeletal disorders with female gender and advanced age, there is no consensus about its association with functional capacity and the use of health services. Conclusion: It was also observed that alcoholism, smoking, physical inactivity, obesity and systemic arterial hypertension are associated with musculoskeletal disorders in the elderly. The high prevalences identified refer to the importance of planning health actions that aim to prepare professionals and public health services to serve this growing portion of the population.
\end{abstract}

Keywords: Aged, Musculoskeletal Diseases, Prevalence 


\section{INTRODUÇÃO}

O envelhecimento populacional, apesar de estabelecer-se mundialmente, ocorreu mais rapidamente nos países em desenvolvimento, entre eles o Brasil, quando comparados aos desenvolvidos, dificultando a reorganização da sociedade e do sistema de saúde para suporte e cuidado as demandas desta população. ${ }^{1,2}$

Linearmente, com o aumento da população idosa há também um crescimento na prevalência de doenças crônicas comuns desta população. ${ }^{1} \mathrm{O}$ processo de envelhecimento é caracterizado por mudanças fisiológicas naturais, que podem levar ao aparecimento de doenças crônicas, dentre elas as alterações ocorridas no sistema músculo-esquelético, que por vezes podem ocasionar distúrbios e dor nos idosos. ${ }^{1}$

Evidência demonstra que os distúrbios osteomusculares são as principais causas de incapacidade relacionada à diminuição da mobilidade, principalmente, na faixa etária 60 anos ou mais. ${ }^{2}$ Dentre os distúrbios osteomusculares, os mais prevalentes são a osteoartrite ${ }^{3-5}$ e a artrite..$^{1,2,6-8}$ As incapacidades podem comprometer a realização de atividades da vida diária, eventualmente, ocasionar dependência e institucionalização, influenciar na autopercepção de saúde e qualidade de vida, e implicar em aumento de custos para os serviços. ${ }^{7}$

Como fatores associados aos distúrbios osteomusculares estão os intrínsecos: sexo feminino, idade avançada, cor branca, deficiência hormonal, presença de comorbidades, fatores genéticos, fragilidade ou histórico de fratura prévia, fraqueza de membros inferiores, déficit de equilíbrio e polifarmácia; e os extrínsecos: baixo peso corpóreo, sedentarismo, tempo de exposição ao tabaco, uso abusivo de álcool. ${ }^{9,10}$

Uma das principais queixas dos idosos com distúrbios osteomusculares crônicos é a dor, causando impacto na qualidade de vida, independência e participação social do idoso. ${ }^{2,11,12} \mathrm{O}$ tratamento da dor caracteriza-se como um desafio para os profissionais e serviços de saúde, pois independente do diagnóstico de distúrbios osteomusculares, a percepção do idoso diante das consequências deste sintoma para a sua vida deve ser ponderado. ${ }^{13}$

\section{OBJETIVO}

Considerando o aumento expressivo da população idosa e da prevalência de disturbios ostemusculares, a presente revisão busca descrever a prevalência de distúrbios osteomusculares e fatores associados na população idosa.

\section{MÉTODO}

Para a seleção dos artigos, efetuou-se busca nas bases de dados Library of Medicine (PUBMED), Scientific Eletronic Library Online (SciELO) Centro Latino-Americano e do Caribe de Informações em Ciências da Saúde (LILACS), e WEB OF SCIENCE durante o mês de agosto de 2018. Os descritores utilizados para a seleção dos estudos foram determinados com base nos Descritores em Ciências da Saúde (DeCS) e nos Medical Subject Headings (MeSH Terms).

Nas bases de dados foram utilizados os descritores em inglês, selecionando-se os idiomas português, inglês e espanhol, sem definição de limite de tempo para a busca.

$\mathrm{Na}$ base de dados PUBMED utilizou-se somente MeSHTerms para busca controlada e foi adicionado um filtro de "humanos" e de idade "middle aged 45-64 years" e "aged 65 + years" para que fosse incluído apenas o público alvo de interesse do estudo.

Foram utilizados os descritores "elderly", "musculoskeletal diseases" e "prevalence" empregando o operador boleano AND entre eles, utilizando-se da mesma estratégia para seleção de artigos nas demais bases de dados de acordo com suas especificidades

A revisão sistemática foi realizada por pares seguindo as recomendações do consenso PRISMA, norteada pela seguinte questão: Qual a prevalência de distúrbios osteomusculares e fatores associados na população idosa?

Em todas as bases de dados foram selecionados artigos de prevalência, estudos realizados com idosos com 60 anos ou mais de idade, e que abordassem os distúrbios osteomusculares.

Os motivos de exclusão, durante as leituras dos títulos, resumos e artigos na íntegra, foram não ter relação com o objetivo desta revisão ou abordarem população específica, como por exemplo: idosos institucionalizados; idosos com síndromes; amostra fora da idade de 60 anos ou mais; doenças específicas como HIV, sífilis, lúpus, distúrbios renais ou cardiovasculares, entre outras; idosos com distúrbios cognitivos ou urinários; pacientes com problemas dentários; trabalhadores e grupos em uso de medicamentos.

\section{RESULTADOS}

Ao realizar as buscas, inicialmente identificou-se 6.290 artigos. Destes, foram excluídos 19 duplicatas, ficando com 6.271 artigos (6.101 da PUBMED, 5 da SciELO, 29 da LILACS e 136 da WEB OF SCIENCE) para a leitura dos títulos. Após a leitura dos títulos no Software Mendeley, foram selecionados 132 artigos para a leitura dos resumos, após foram selecionados 60 artigos para a leitura na íntegra.

Ao final foram selecionados 21 artigos, excluindo-se aqueles que não respondiam à questão norteadora e não tinham um desfecho equivalente à proposta dessa revisão (Figura 1). Dos artigos que constituiram essa revisão, 19 artigos foram publicados em inglês e apenas dois em português, com datas de publicação entre 1991 e 2018. A maioria dos artigos foram produzidos no Brasil (6), seguido de México (2), Dinamarca (2) e Estados Unidos (2). Os países que apresentaram apenas uma publicação para o tema foram: Espanha, Reino Unido, Nigéria, Finlândia, China, Japão, Turquia, Suécia e Holanda. Quanto ao delineamento, 19 artigos são estudos transversais e apenas dois estudos de coorte.

Aproximadamente metade dos artigos (10) selecionaram idosos acima de 60 anos para o estudo, sendo que seis eram brasileiros e os demais do Japão, Dinamarca, Nigéria e México. Os demais estudos selecionaram idosos em idades que compreendiam entre 64 e 85 anos de idade.

No Quadro 1 apresenta-se a síntese dos artigos que compõem esta revisão destacando-se: autor(es)/ano, título, tipo de estudo, ferramenta de coleta, local/amostra, principais achados referentes aos distúrbios osteomusculares e respectivos fatores associados. 
Entre os estudos selecionados observou-se uma grande variabilidade na prevalência de disturbios osteomusculares em idosos, variando de $0,6 \% 7$ a $72,1 \%,{ }^{14}$ com impacto variável nas funções física e psicológica do sujeito. ${ }^{5}$

Os instrumentos utilizados para identificar a prevalência de disturbios ostemusculares foram variados, não existindo consenso em relação às formas de avaliação. Alguns autores mensuraram os distúrbios utilizando escalas de incapacidades, 5,8,15,16 outros aplicaram questionário estruturado, composto por questões demográficas, socioeconômicas, comportamentais, de estilo de vida, presença de doenças e consumo alimentar. ${ }^{1-3,6,7,14,16,17,18,19,21-23}$

As características comportamentais e de estilo de vida estão entre aquelas que mais influenciaram a saúde dos idosos, destacando-se a dieta pouco saudável, a inatividade física, o tabagismo e o abuso do álcool. ${ }^{24}$

Dentre os distúrbios ostemusculares mais citados pelos autores estão artrite (33,3\%), dor nas costas $(28,5 \%)$, e osteoartrite $(23,8 \%)$ e osteoporose $(14,2 \%)$. Os fatores associados mais evidenciados foram o sexo feminino ${ }^{1-8,14}$ 18,20,21,23,25-27 podendo chegar ao dobro da prevalência quando comparado com o sexo masculino; 1,2 vezes maior em idosos com idade mais avançada ${ }^{4,7,14,21}$ e um quinto maior em idosos com obesidade. ${ }^{3,4,19,22}$ A a raça branca, ${ }^{4,23}$ apesar de uma associação menos evidente, pode apresentar-se como fator de proteção dependendo da localização da dor.

\section{DISCUSSÃO}

Entre os distúrbios osteomusculares mais prevalentes encontra-se a artrite atingindo $65,4 \%,{ }^{4}$ seguida de dor nas costas $56,3 \%^{8}$ e osteoartrite (quadril, joelhos, mãos, generalizada ou degenerativa) com $51,9 \% .{ }^{4}$ Kaplan et al. ${ }^{28}$ apontam que a prevalência de artrite aumenta acentuadamente após os 45 anos, e quase metade de todas as pessoas com esse distúrbio tem 65 anos de idade ou mais, com projeção de elevação nas próximas décadas, por exemplo, nos Estados Unidos aumentando de 15,0\% em 1991 para 18,2\% em $2020 .^{28}$

Evidências da literatura destacam que o diagnóstico de osteoartrite está fortemente associado ao envelhecimento, indepedentemente da localização (pequenas articulações ou articulações de maior suporte de peso) ou do sexo. Dados recentes indicam um aumento de $30,0 \%$ na prevalência dessa condição em uma década, e sugerem que esse aumento devese a uma combinação de fatores, como o envelhecimento da população e a prevalência crescente de fatores de risco, tais como sexo feminino, menor escolaridade, tabagismo, idade mais avançada. ${ }^{13}$

A dor musculoesquelética foi observada em $72,1 \%$ dos idosos, ${ }^{14}$ enquanto a dor nas costas varia de $25,0 \%{ }^{16}$ à $56,3 \%{ }^{8}$ Os idosos que referiram dor musculoesquelética relataram autoavaliação de saúde regular e ruim, já aqueles sem dor relataram como excelente - muito bom - boa saúde, ${ }^{14}$ demonstrando perda da qualidade de vida naqueles com dor.

Estudos evidenciam que idosos com osteoartrite que fazem fisioterapia aquática ou terrestre tem melhora significativa no quesito subir, descer escadas e caminhar, o que beneficia no desempenho do dia a dia do idoso. A capacidade funcional dos

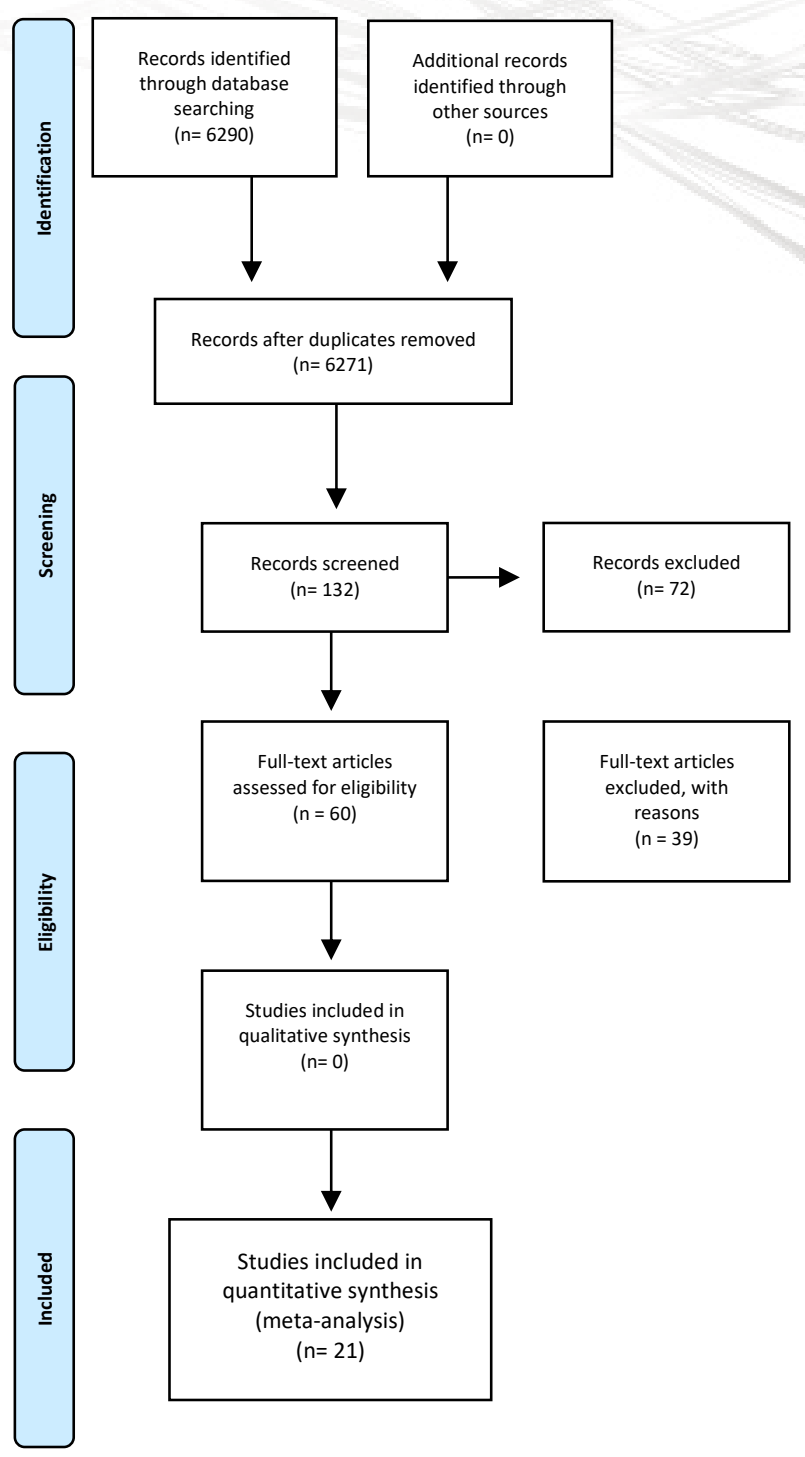

Figura 1. Fluxograma das etapas do processo de revisão sistemática para a seleção de artigos

idosos está relacionada com a independência dos mesmos para realização as suas atividades de vida diária, e a intervenção terapêutica contribui para melhora da qualidade de vida dos idosos. ${ }^{29,30}$

As alterações corporais que acompanham os indivíduos no decorrer dos anos e o aparecimento de doenças crônicas levam a um desgaste nos componentes de sustentação da coluna, modificando a anatomia e a fisiologia, o que pode acarretar na ocorrência de dor nas costas. ${ }^{31}$

Embora os distúrbios osteomusculares acometam ambos os sexos, $90,5 \%$ dos artigos (19) verificaram que estes são mais prevalentes em mulheres. ${ }^{1-8,14-18,20,21,23,25-27}$ Este achado pode resultar da mais pronunciada perda muscular no sexo feminino, de aproximadamente $5,0 \%$ a cada década até os 50 anos, e após, $10,0 \%$ a cada década até os 80 anos, devido as próprias características fisiológicas femininas, que compreendem ter menos músculo e massa óssea e a menopausa, em que há perda de sais minerais e diminuição dos níveis de estrogênio, hormônio relacionado às estimulações das atividades dos osteoblastos. ${ }^{32-34}$ 
Quadro 1. Síntese dos artigos selecionados na revisão sistemática

\begin{tabular}{|c|c|c|c|c|c|}
\hline Autor(es) / Ano & $\begin{array}{l}\text { Tipo de } \\
\text { Estudo }\end{array}$ & $\begin{array}{l}\text { Ferramenta } \\
\text { de Coleta }\end{array}$ & Local / Amostra & Principais achados de Distúrbios Osteomusculares & Fatores Associados \\
\hline $\begin{array}{l}\text { Herr et al. }{ }^{25} \\
1991\end{array}$ & Transversal & Entrevista & $\begin{array}{l}\text { lowa (EUA) / } 3.097 \text { idosos } \\
\text { rurais } 65 \text { anos ou mais }\end{array}$ & $\begin{array}{l}64,0 \% \text { relataram ter dores nas pernas no ano anterior à } \\
\text { entrevista } \\
21,0 \% \text { relatou dor nas pernas enquanto caminha } \\
56,0 \% \text { dor ou cãibras à noite } \\
14,0 \% \text { relataram as duas formas de dor }\end{array}$ & Sexo feminino \\
\hline $\begin{array}{l}\text { Van } \\
\text { Schaardenburg et } \\
\text { al. } .^{5} \\
1994\end{array}$ & Transversal & $\begin{array}{l}\text { Visitas } \\
\text { domiciliares } \\
\text { com exames } \\
\text { médicos }\end{array}$ & $\begin{array}{l}\text { Holanda/ } 73 \text { mulheres e } 32 \\
\text { homens com mais de } 85 \text { anos } \\
\text { de idade }\end{array}$ & $\begin{array}{l}57,0 \% \text { dos entrevistados relataram dor nas costas ou dor nas } \\
\text { articulações, ou ambos ( } 62,0 \% \text { nas mulheres / } 47,0 \% \text { nos } \\
\text { homens) }\end{array}$ & Sexo feminino \\
\hline $\begin{array}{l}\text { Grimby et al. }{ }^{20} \\
1999\end{array}$ & Transversal & $\begin{array}{l}\text { Questionário } \\
\text { sobre auto } \\
\text { avaliação de } \\
\text { saúde }\end{array}$ & $\begin{array}{l}\text { Estocolmo / } 1.800 \text { idosos com } \\
75 \text { anos ou mais }\end{array}$ & $\begin{array}{l}60 \% \text { relataram problemas com dores músculo - esqueléticas } \\
40 \% \text { dos indivíduos que relataram dor usaram analgésicos }\end{array}$ & Sexo feminino \\
\hline $\begin{array}{l}\text { Al Snih et al. }{ }^{18} \\
2001\end{array}$ & Transversal & $\begin{array}{l}\text { Questionário } \\
\text { estruturado }\end{array}$ & $\begin{array}{l}\text { México / } 2.167 \text { homens e } \\
\text { mulheres mexicanos não- } \\
\text { institucionalizados de } 65 \\
\text { anos }\end{array}$ & $\begin{array}{l}31,9 \% \text { de dor ( } 37,7 \% \text { nas mulheres e } 24,0 \% \text { nos homens). Os } \\
\text { locais de dor mais prevalentes foram joelhos }(14,7 \%) \text { e tornozelo } \\
\text { / pés }(12,1 \%)\end{array}$ & Sexo feminino \\
\hline $\begin{array}{l}\text { Hartvigsen et al. }{ }^{16} \\
2003\end{array}$ & Transversal & $\begin{array}{l}\text { Escala de } \\
\text { incapacidades }\end{array}$ & $\begin{array}{l}\text { Dinamarca / } 4.486 \text { gêmeos } \\
\text { dinamarqueses entre } 70 \text { e } \\
102 \text { anos }\end{array}$ & $25,0 \%$ de dor nas costas em um mês & $\begin{array}{l}\text { Sexo feminino, hipertensão } \\
\text { arterial sistêmica }\end{array}$ \\
\hline $\begin{array}{l}\text { Vogt et al. }{ }^{23} \\
2003\end{array}$ & Transversal & $\begin{array}{l}\text { Questionário } \\
\text { estruturado }\end{array}$ & $\begin{array}{l}\text { Pittsburgh e Memphis / } 335 \\
\text { homens e mulheres, idades } \\
\text { entre } 70 \text { a } 79 \text { anos, que } \\
\text { participam do Estudo de } \\
\text { Saúde, Envelhecimento e } \\
\text { Composição Corporal (Health } \\
\text { ABC) }\end{array}$ & $\begin{array}{l}11,9 \% \text { relataram dor cervical com um mês ou mais de duração e } \\
18,9 \% \text { dor no ombro } \\
\text { As mulheres brancas tiveram a maior prevalência de dor cervical } \\
(15,4 \%) \text { e a maior prevalência de dor no ombro }(24,3 \%)\end{array}$ & Sexo feminino, raça branca \\
\hline $\begin{array}{l}\text { Cavlak et al. }{ }^{14} \\
2009\end{array}$ & Transversal & $\begin{array}{l}\text { Questionário } \\
\text { estruturado }\end{array}$ & $\begin{array}{l}\text { Turquia / } 900 \text { homens e } \\
\text { mulheres ( } 65 \text { anos ou mais) }\end{array}$ & $\begin{array}{l}72,1 \% \text { referiram dor musculoesquelética ( } 85,5 \% \text { nas mulheres e } \\
61,8 \% \text { nos homens) } \\
\text { A maioria dos indivíduos com dor musculoesquelética relatou } \\
\text { autoavaliação de saúde regular e ruim, enquanto os sem dor } \\
\text { auto avaliaram a saúde como boa, muito boa ou excelente } \\
\text { A dor das extremidades inferiores foi mais comum para ambos } \\
\text { os sexos }\end{array}$ & $\begin{array}{l}\text { Sexo feminino, idade mais } \\
\text { avançada }\end{array}$ \\
\hline $\begin{array}{l}\text { Muraki et al. }{ }^{7} \\
2009\end{array}$ & Transversal & $\begin{array}{l}\text { Questionário } \\
\text { estruturado }\end{array}$ & $\begin{array}{l}\text { Itabashi-ku, comunidade } \\
\text { urbana em Tóquio / } 2.282 \\
\text { idosos com } 60 \text { anos ou mais }\end{array}$ & 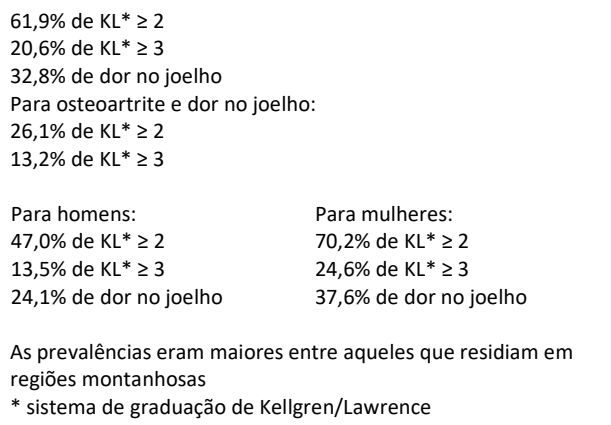 & $\begin{array}{l}\text { Sexo feminino, idade mais } \\
\text { avançada, IMC alto, residência } \\
\text { rural }\end{array}$ \\
\hline $\begin{array}{l}\text { Woo et al. }{ }^{17} \\
2009\end{array}$ & Coorte & $\begin{array}{l}\text { Questionário } \\
\text { estruturado }\end{array}$ & $\begin{array}{l}\text { Hong Kong / } 4.000 \text { homens e } \\
\text { mulheres, com } 65 \text { anos ou } \\
\text { mais }\end{array}$ & $\begin{array}{l}48,0 \% \text { de dor nas costas } \\
31,0 \% \text { de dor no joelho } \\
22,5 \% \text { de dor no pescoço } \\
8,9 \% \text { de dor no quadril } \\
\text { Sendo, que prevalência de dor em todos os locais foi quase o } \\
\text { dobro entre as mulheres comparadas com os homens } \\
\text { A gravidade, frequencia e incomodo nos últimos } 12 \text { meses antes } \\
\text { do estudo foi maior entre as mulheres (entre } 6,0 \% \text { e } 9,6 \% \text { do } \\
\text { ciática e } 4,7 \% \text { dor cervical) } \\
\text { A dor nas costas afetou o escore de atividade física }\end{array}$ & $\begin{array}{l}\text { Sexo feminino: } \\
\text { - diminuição mais acentuada } \\
\text { da massa muscular com o } \\
\text { aumento da idade. } \\
\text { - menor limiar para dor } \\
\text { - declíneo acentuado e precoce } \\
\text { da densidade mineral óssea } \\
\text { após a menopausa com } \\
\text { subsequente aumento de } \\
\text { osteoporose e fraturas } \\
\text { - deficiência de estrogênio no } \\
\text { desenvolvimento da } \\
\text { osteoartrite } \\
\text { Condição socioeconômica mais } \\
\text { baixa: } \\
\text { questões emocionais }\end{array}$ \\
\hline $\begin{array}{l}\text { Frost et al. }{ }^{19} \\
2012\end{array}$ & Transversal & $\begin{array}{l}\text { Questionário } \\
\text {, entrevista } \\
\text { por telefone, } \\
\text { exame físico } \\
\text { e registros } \\
\text { médicos do } \\
\text { hospital }\end{array}$ & $\begin{array}{l}\text { Dinamarca / } 600 \text { homens } \\
\text { entre } 60-74 \text { anos de idade }\end{array}$ & $\begin{array}{l}\text { Osteoartrite, artrite reumatoide ou qualquer outra doença } \\
\text { musculoesquelética: } \\
\text { - } 7,8 \% \text { de auto-relato } \\
\text { - 20,8\% de avaliação de clínica }\end{array}$ & -- \\
\hline
\end{tabular}


Quadro 1. Síntese dos artigos selecionados na revisão sistemática

(Continuação)

\begin{tabular}{|c|c|c|c|c|c|c|}
\hline Autor(es) / Ano & $\begin{array}{l}\text { Tipo de } \\
\text { Estudo }\end{array}$ & $\begin{array}{c}\text { Ferramenta } \\
\text { de Coleta }\end{array}$ & Local / Amostra & \multicolumn{2}{|c|}{ Principais achados de Distúrbios Osteomusculares } & Fatores Associados \\
\hline $\begin{array}{l}\text { Duncan et al. }{ }^{4} \\
2011\end{array}$ & Coorte & $\begin{array}{l}\text { Revisão de } \\
\text { registros e } \\
\text { revisão por } \\
\text { enfermeiros } \\
\text { no domicílio } \\
\text { de avaliação } \\
\text { de saúde }\end{array}$ & $\begin{array}{l}\text { Newcastle, Reino Unido / } \\
1.040 \text { idosos com } 85 \text { anos de } \\
\text { idade }\end{array}$ & \multicolumn{2}{|c|}{$\begin{array}{l}\text { Artrite ao longo da vida: } \\
65,4 \% \text { (IC } 95 \%: 62,5-68,3 \text { ) } \\
-69,1 \% \text { em mulheres } \\
-58,8 \% \text { em homens } \\
\text { Osteoartrite: } \\
51,9 \% \text { (IC } 95 \%: 48,8-54,9 \text { ) } \\
-57,1 \% \text { em mulheres } \\
-42,5 \% \text { em homens } \\
63,1 \% \text { de dor nas articulações no último mês (nas } 11 \text { áreas } \\
\text { avaliadas) }\end{array}$} & $\begin{array}{l}\text { Sexo feminino, raça branca, } \\
\text { idade avançada, tabagismo, } \\
\text { inatividade física e obesidade. } \\
\text { Não apresenta explicações } \\
\text { para as associações } \\
\text { encontradas }\end{array}$ \\
\hline $\begin{array}{l}\text { Blay et al. } \\
2012\end{array}$ & Transversal & $\begin{array}{l}\text { Questionário } \\
\text { estruturado }\end{array}$ & $\begin{array}{l}\text { Rio Grande do Sul / } 6.963 \\
\text { idosos com } 60 \text { anos ou mais }\end{array}$ & \multicolumn{2}{|c|}{$\begin{array}{l}43,1 \% \text { de artrite }(75,8 \% \text { em mulheres e } 24,2 \% \text { em homens }) \\
\text { A artrite foi associada à redução das chances de acidente } \\
\text { vascular cerebral e a escolaridade } \geq 4 \text { anos é fator de proteção } \\
\text { para a artrite }\end{array}$} & $\begin{array}{l}\text { Sexo feminino, menor } \\
\text { escolaridade (<4 anos), baixa } \\
\text { renda, empregado, } \\
\text { comportamentos de saúde } \\
\text { (participação em atividades } \\
\text { sociais), limitações funcionais e } \\
\text { condições de saúde (ter 1-2 } \\
\text { limitações, hipertensão, AVC, } \\
\text { varicosidades, osteoporose, } \\
\text { bronquite, infecção urinária, } \\
\text { problemas renais, dor de } \\
\text { cabeça, distúrbios } \\
\text { gastrointestinais, mais de três } \\
\text { sintomas de depressão) }\end{array}$ \\
\hline $\begin{array}{l}\text { Abegunde } \mathrm{K}, \\
\text { Owoaje } \mathrm{E}^{3} \\
2013\end{array}$ & $\begin{array}{l}\text { Transversal } \\
\text { comparativo }\end{array}$ & $\begin{array}{l}\text { Questionário } \\
\text { estruturado }\end{array}$ & $\begin{array}{l}\text { Iseyin (urbana) e llua (rural) } \\
\text { comunidades do Estado de } \\
\text { Oyo, no sudoeste da Nigéria } \\
\text { / } 630 \text { idosos com } 60 \text { anos ou } \\
\text { mais }\end{array}$ & \multicolumn{2}{|c|}{$\begin{array}{l}5,9 \% \text { de osteoartrite }(8,5 \% \text { em população urbana e } 3,2 \% \text { em } \\
\text { rural - } p=0,004) \\
\text { Dor lombar: } \\
41,8 \% \text { pop. urbana } \\
38,5 \% \text { pop. rural } \\
22,0 \% \text { da pop. urbana e rural buscaram o serviço de saúde por } \\
\text { problemas musculo - esqueléticos }\end{array}$} & $\begin{array}{l}\text { Sexo feminino em população } \\
\text { urbana e obesidade para } \\
\text { ambas populações (urbana e } \\
\text { rural) }\end{array}$ \\
\hline $\begin{array}{l}\text { Falsarella et al. }{ }^{15} \\
2013\end{array}$ & Transversa & $\begin{array}{l}\text { Questionário } \\
\text { estruturado }\end{array}$ & $\begin{array}{l}\text { São Paulo, Brasil / } 2.209 \\
\text { idosos } 60 \text { anos }\end{array}$ & \multicolumn{2}{|c|}{$\begin{array}{l}22,7 \% \text { de reumatismo ( } 73,5 \% \text { em mulheres e } 26,5 \% \text { em homens) } \\
45,6 \% \text { de sintomas articulares crônicos }(66,5 \% \text { em mulheres e } \\
33,5 \% \text { em homens) }\end{array}$} & $\begin{array}{l}\text { O reumatismo associou-se ao } \\
\text { sexo feminino, renda familiar } \\
\text { baixa, doença cardiovascular, } \\
\text { catarata, uso de } \\
\text { medicamentos, baixa } \\
\text { qualidade de vida } \\
\text { Os sintomas articulares } \\
\text { crônicos associaram-se ao sexo } \\
\text { feminino, ao índice de massa } \\
\text { corporal (quanto maior o IMC } \\
\text { maior a RO), ao uso de } \\
\text { medicamentos, a baixa } \\
\text { capacidade funcional, a saúde } \\
\text { geral e a dor }\end{array}$ \\
\hline $\begin{array}{l}\text { Nunes et al. }{ }^{1} \\
2015\end{array}$ & Transversal & $\begin{array}{l}\text { Questionário } \\
\text { estruturado }\end{array}$ & $\begin{array}{l}\text { Bagé, RS, Brasil / } 1.593 \text { idosos } \\
60 \text { anos ou mais }\end{array}$ & \multicolumn{2}{|c|}{$\begin{array}{l}37,4 \% \text { de doença da coluna vertebral } \\
23,6 \% \text { dos idosos apresentaram hipertensão arterial sistêmica e } \\
\text { doença da coluna vertebral } \\
10,6 \% \text { dos idosos apresentaram hipertensão arterial sistêmica e } \\
\text { reumatismo / artrite / artrose e doença da coluna vertebral }\end{array}$} & Hipertensão arterial sistêmica \\
\hline $\begin{array}{l}\text { Foguet-Boreu et } \\
\text { al. }{ }^{26} \\
2015\end{array}$ & Transversal & $\begin{array}{l}\text { Registros } \\
\text { médicos do } \\
\text { sistema de } \\
\text { informação } \\
\text { da Atenção } \\
\text { Primária na } \\
\text { Espanha }\end{array}$ & $\begin{array}{l}\text { Catalonia, Espanha / } 343.358 \\
\text { idosos com mais de } 64 \text { anos } \\
\text { de idade registrados no } \\
\text { registro de cuidados }\end{array}$ & $\begin{array}{l}\text { Artrite em Mulheres } \\
65-79 \text { anos: } \\
41,3 \% \text { no estrato } \\
45,3 \% \text { no grupo } \\
\text { Artrite em Homens } \\
65-79 \text { anos: } \\
22,7 \% \text { no estrato } \\
36,7 \% \text { no grupo }\end{array}$ & $\begin{array}{l}\geq 80 \text { anos: } \\
46,9 \% \text { no estrato } \\
50,0 \% \text { no grupo } \\
\geq 80 \text { anos: } \\
30,9 \% \text { no estrato } \\
32,2 \% \text { no grupo }\end{array}$ & Sexo feminino \\
\hline $\begin{array}{l}\text { Silveira et al. }{ }^{22} \\
2016\end{array}$ & Transversal & $\begin{array}{l}\text { Questionário } \\
\text { estruturado }\end{array}$ & $\begin{array}{l}\text { Goiânia, GO, Brasil / } 418 \\
\text { idosos ( } \geq 60 \text { anos) atendidos } \\
\text { na rede de atenção básica }\end{array}$ & \multicolumn{2}{|c|}{$33,7 \%$ doenças do sistema osteomuscular } & Obesidade \\
\hline $\begin{array}{l}\text { Yokota et al. } \\
2016\end{array}$ & Transversal & $\begin{array}{l}\text { Escala de } \\
\text { incapacidades }\end{array}$ & $\begin{array}{l}\text { Brasil / } 10.290 \text { Indivíduos } \\
\text { com } 60 \text { anos ou mais que } \\
\text { participaram da Pesquisa } \\
\text { Nacional de Saúde - PNS } \\
\text { realizada no Brasil em } 2013\end{array}$ & $\begin{array}{l}\text { Artrite } \\
10,1 \% \text { em homens (IC } 8,3- \\
11,8 \% \text { ) } \\
22,2 \% \text { em mulheres (IC } \\
20,5 \%-23,8 \% \text { ) } \\
\text { Incapacidade se deve: } \\
30,0 \% \text { em homens (AVC, do } \\
20,0 \% \text { em mulheres (diabet }\end{array}$ & $\begin{array}{l}\text { Dor nas costas } \\
24,7 \% \text { em homens (IC } \\
22,3 \%-26,9 \%) \\
31,6 \% \text { em mulheres }(29,7 \%- \\
33,5 \%) \\
\text { costas e artrite) } \\
\text { eença cardíaca e artrite) }\end{array}$ & Sexo feminino \\
\hline $\begin{array}{l}\text { Melo et al. }{ }^{2} \\
2017\end{array}$ & Transversal & $\begin{array}{l}\text { Questionário } \\
\text { estruturado }\end{array}$ & $\begin{array}{l}\text { Goiânia, GO, Brasil / } 934 \\
\text { idosos com } 60 \text { anos ou mais }\end{array}$ & $\begin{array}{l}39,1 \% \text { doenças musculoesq } \\
-24,6 \% \text { osteoporose (IC } 21, \\
-2,7 \% \text { artrose (IC } 1,7 \%-3,9 \% \\
-1,2 \% \text { reumatismo (IC } 0,5 \% \\
-0,6 \% \text { artrite (IC } 0,2 \%-1,4 \% \text { ) } \\
-9,8 \% \text { outras DME (IC } 8,2 \%-\end{array}$ & $\begin{array}{l}\text { icas - DME (IC 35,9\% - 42,3\%) } \\
7,5 \%) \\
\%)\end{array}$ & $\begin{array}{l}\text { Sexo feminino, } \\
\text { idade } \geq 70 \text { anos, } \\
\text { autoavaliação de saúde ruim / } \\
\text { muito ruim, } \\
\text { presença de dor }\end{array}$ \\
\hline $\begin{array}{l}\text { Islas-Granillo H } \\
\text { et al. }{ }^{27}\end{array}$ & Transversal & $\begin{array}{l}\text { Questionário } \\
\text { estruturado }\end{array}$ & $\begin{array}{l}\text { México / } 139 \text { idosos com } 60 \\
\text { anos ou mais }\end{array}$ & $19,4 \%$ de distúrbios osteom & lares & $\begin{array}{l}19,4 \% \text { de distúrbios } \\
\text { osteomusculares }\end{array}$ \\
\hline
\end{tabular}


Outro fator associado aos distúrbios osteomusculares foi a idade mais avançada (acima de 75 anos) observada em quatro estudos. ${ }^{4,5,14,21}$ Apesar da prevalência de problemas crônicos de coluna aumentar com a idade, a relação não é linear e se estabiliza por volta dos 60 anos, sendo mais importante o impacto da gravidade das limitações e não o aumento da prevalência. ${ }^{35,36}$ Essas limitações se devem ao prejuízo da função muscular e a consequente participação da função motora que, associados ao processo de envelhecimento, influenciam na qualidade de vida dos idosos, culminando na incapacidade funcional, entendida como a inabilidade ou a dificuldade de realizar tarefas que fazem parte do cotidiano do ser humano como caminhar. ${ }^{37,38}$

O tabagismo, ${ }^{3,4,20}$ o alcoolismo, ${ }^{3,20}$ a inatividade física e a obesidade $\mathrm{o}^{3,4,19}$ estiveram associados aos distúrbios osteomusculares. A obesidade também esteve associada à hipertensão arterial sistemica ${ }^{1,16,19}$ e ao estilo de vida, promovendo sobrecarga das estruturas articulares da coluna lombossacra, o que predispõe a degeneração. ${ }^{32}$

Quando citadas as características sobre escolaridade como indicador de nível socioeconômico, constatou-se que o baixo nível educacional pode contribuir diretamente para que as pessoas não entendam adequadamente sobre o cuidado de sua saúde, o que colaboraria para a manifestação de um maior número de doenças nesses indivíduos. ${ }^{39} \mathrm{Na}$ população em geral grupos com menor escolaridade possuem piores condições de saúde $^{40}$ e realizam menos consultas médicas do que aqueles com mais anos de estudo. ${ }^{41}$ Os serviços de saúde que utilizassem programas de promoção à saúde demonstrariam maior interesse neste grupo da população, os idosos.

Sendo que se sabe que dor nas costas é responsável por uma das maiores demandas do serviço de saúde e dos ambulatórios de fisioterapia, e também que a diminuição da capacidade funcional de idosos leva à uma redução das suas atividades de vida diária, o que acarreta em menos independência e menor participação dos idosos na comunidade. ${ }^{30,31}$ Há um déficit de estudos que tragam mais elucidada a relação entre o uso dos serviços de saúde e a manutenção da capacidade funcional da população idosa.

O modelo proposto por Dahlgren e Whitehead auxilia na compreensão do impacto dos determinantes sociais na saúde do idoso, identificando as interações entre os níveis de condições sociais e as desigualdades em saúde, desde o nível individual até as condições econômicas, culturais e ambientais que se sobressaem na sociedade. Individualmente, consideram-se a idade, o sexo e os fatores genéticos e no nível superior estão os fatores associados ao comportamento e estilo de vida, que cooperam para a exposição diferencial a fatores de risco como o tabagismo e sedentarismo. ${ }^{24}$

Apesar da pesente revisão ser realizada por pares seguindo as recomendações do consenso PRISMA avaliando a qualidade dos estudos deve-se observar os dados com parcimônia devido ao riscos de viés de seleção, mensuração e informação, pois os estudos incluidos abordam diferentes populações (americanos, europeus, asiaticos), desenhos de estudo (transversal e coorte), instrumentos (questionário estruturado, escalas de incapacidade, exame clínico, entre outros), desfechos, tempo recordatório, seleção dos participantes para estudo, além do viés de publicação. ${ }^{42,43}$

\section{CONCLUSÃO}

As prevalências elevadas de distúrbios osteomusculares observadas nos estudos remetem a necessidade de planejar ações em saúde, visando preparar os profissionais e os serviços públicos de saúde para atender esta parcela crescente da população, tornando-se essencial o desenvolvimento de políticas de saúde que incorporem planos estratégicos com enfase nos distúrbios osteomusculares e suas consequências.

Considerando os dados encontrados nesta revisão, verificase que apesar da literatura consolidar a associação entre os distúrbios osteomusculares com determinadas características dos idosos, como sexo feminino e idade avançada, não há consenso acerca da capacidade funcional e da utilização de serviços de saúde.

Além disso, há uma grande variabilidade nas prevalências e nos instrumentos utilizados para avaliar os disturbios ostemusculares, evidenciando-se a necessidade de desenvolver estudos sobre a temática, definir a forma mais adequada de avaliar a presença de distúrbios osteomusculares buscando compreender seus fatores de determinantes e condicionantes a fim de desenvolver ações de promoção da saúde, prevenção de agravos e manutenção de qualidade de vida.

\section{REFERÊNCIAS}

1. Nunes BP, Thume E, Facchini LA. Multimorbidity in older adults: magnitude and challenges for the Brazilian health system. BMC Public Health. 2015;15:1172. Doi: http://dx.doi.org/10.1186/s12889-015-2505-8

2. Melo ACF, Nakatani AYK, Pereira LV, Menezes RL, Pagotto V. Prevalência de doenças musculoesqueléticas autorreferidas segundo variáveis demográficas e de saúde: estudo transversal de idosos de Goiânia/GO. Cad Saúde Colet. 2017;25(2):138-43. Doi: https://doi.org/10.1590/1414$\underline{462 \times 201700010274}$

3. Abegunde KA, Owoaje ET. Health problems and associated risk factors in selected urban and rural elderly population groups of South-West Nigeria. Ann Afr Med. 2013;12(2):907. Doi: http://dx.doi.org/10.4103/1596-3519.112398

4. Duncan R, Francis RM, Collerton J, Davies K, Jagger C, Kingston $A$, et al. Prevalence of arthritis and joint pain in the oldest old: findings from the Newcastle $85+$ study. Age Ageing. 2011;40(6):752. Doi: http://dx.doi.org/10.1093/ageing/afr105

5. Van Schaardenburg D, Van den Brande KJ, Ligthart GJ, Breedveld FC, Hazes JM. Musculoskeletal disorders and disability in persons aged 85 and over: a community survey. Ann Rheum Dis. 1994;53(12):807-11. Doi: http://dx.doi.org/10.1136/ard.53.12.807

6. Blay SL, Fillenbaum GG, Andreoli SB, Gastal FL. Prevalence and concomitants of arthritis in the elderly in Rio Grande do Sul, Brazil. PLoS One. 2012;7(9):e45418. DOI: http://dx.doi.org/10.1371/journal.pone.0045418

7. Muraki S, Akune T, Oka H, Mabuchi A, En-Yo Y, Yoshida M, et al. Association of occupational activity with radiographic knee osteoarthritis and lumbar spondylosis in elderly patients of population-based cohorts: a large-scale population-based study. Arthritis Rheum. 2009;61(6):77986. Doi: http://dx.doi.org/10.1016/j.joca.2009.04.005 
8. Yokota RTC, Van der Heyden J, Nusselder WJ, Robine J-M, Tafforeau J, Deboosere $P$, et al. Impact of chronic conditions and multimorbidity on the disability burden in the older population in Belgium. J Gerontol A Biol Sci Med Sci. 2016;71(7):903-9. Doi: http://dx.doi.org/10.1007/s00038 016-0843-7

9. Bello Al, Ababio E, Antwi-Baffoe S, Seidu MA, Adjei DN. Pain, range of motion and activity level as correlates of dynamic balance among elderly people with musculoskeletal disorder. Ghana Med J. 2014;48(4):214-8. Doi: http://dx.doi.org/10.4314/gmj.v48i4.8

10. Curtis E, Litwic A, Cooper C, Dennison E. Determinants of muscle and bone aging. J Cell Physiol. 2015;230(11):2618-25. Doi: http://dx.doi.org/10.1002/jcp.25001

11. Azevedo AL, Silva RA, Tomasi E, Quevedo LA. Doenças crônicas e qualidade de vida na atenção primária à saúde. Cad Saude Publica. 2013;29(9):1774-82. Doi: http://dx.doi.org/10.1590/0102-311X00134812

12. Gokce Kutsal Y, Ozdemir O, Karahan S, Akyol Y, Borman P, Dogan A, et al. Musculoskeletal pain in elderly patients with osteoporosis: a multicenter study. Turk J Phys Med Rehabil. 2012;58(4):263-6. Doi: http://dx.doi.org/10.4274/tftr.04706

13. Miranda VS, Decarvalho VB, Machado LA, Dias JM. Prevalence of chronic musculoskeletal disorders in elderly Brazilians: a systematic review of the literature. BMC Musculoskelet Disord. 2012;13:82. Doi: http://dx.doi.org/10.1186/1471-2474-13-82

14. Cavlak U, Yagci N, Bas Aslan U, Ekici G. A new tool measuring health-related quality of life (HRQOL): the effects of musculoskeletal pain in a group of older Turkish people. Arch Gerontol Geriatr. 2009;49(2):298-303. Doi: http://dx.doi.org/10.1016/j.archger.2008.11.002

15. Falsarella GR, Coimbra IB, Barcelos CC, Costallat LT, Carvalho OM, Coimbra AM. Prevalence and factors associated with rheumatic diseases and chronic joint symptoms in the elderly. Geriatr Gerontol Int. 2013;13(4):1043-50. Doi: http://dx.doi.org/10.1111/ggi.12052

16. Hartvigsen J, Christensen $\mathrm{K}$, Frederiksen H. Back pain remains a common symptom in old age. a population-based study of 4486 Danish twins aged 70-102. Eur Spine J. 2003;12(5):52834. Doi: http://dx.doi.org/10.1007/s00586-003-0542-y

17. Woo J, Leung J, Lau E. Prevalence and correlates of musculoskeletal pain in Chinese elderly and the impact on 4year physical function and quality of life. Public Health. 2009;123(8):549-56.

Doi:

http://dx.doi.org/10.1016/j.puhe.2009.07.006

18. Al Snih S, Markides KS, Ray L, Goodwin JS. Impact of pain on disability among older Mexican Americans. J Gerontol A Biol Sci Med Sci. 2001;56(7):M400-4. Doi: http://dx.doi.org/10.1093/gerona/56.7.m400

19. Frost M, Wraae K, Gudex C, Nielsen T, Brixen K, Hagen C, Andersen $M$. Chronic diseases in elderly men: underreporting and underdiagnosis. Age Ageing. 2012;41(2):177-83.

http://dx.doi.org/10.1093/ageing/afr153

Doi:

20. Grimby C, Fastbom J, Forsell Y, Thorslund M, Claesson CB, Winblad B. Musculoskeletal pain and analgesic therapy in a very old population. Arch Gerontol Geriatr. 1999;29(1):2943. Doi: http://dx.doi.org/10.1016/s0167-4943(99)00021-7

21. Laiho K, Tuomilehto J, Tilvis R. Prevalence of rheumatoid arthritis and musculoskeletal diseases in the elderly population. Rheumatol Int. 2001;20(3):85-7. Doi: http://dx.doi.org/10.1007/s002960000087
22. Silveira EAD, Vieira LL, Jardim TV, Souza JD. Obesity and its association with food consumption, diabetes mellitus, and acute myocardial infarction in the elderly. Arq Bras Cardiol. 2016;107(6):509-517.

http://dx.doi.org/10.5935/abc.20160182

23. Vogt MT, Simonsick EM, Harris TB, Nevitt MC, Kang JD, Rubin SM, et al. Neck and shoulder pain in 70- to 79-year-old men and women: findings from the health, aging and body composition study. Spine J. 2003;3(6):435-41. Doi: http://dx.doi.org/10.1016/s1529-9430(03)00150-5

24. Geib LTC. Determinantes sociais da saúde do idoso. Ciênc Saúde Col. 2012;17(1):123-33. Doi: http://dx.doi.org/10.1590/S1413-81232012000100015

25. Herr KA, Mobily PR, Wallace RB, Chung Y. Leg pain in the rural lowa 65+ population. Prevalence, related factors, and association with functional status. Clin J Pain. 1991;7(2):114 21. Doi: http://dx.doi.org/10.1097/00002508-199106000$\underline{00007}$

26. Foguet-Boreu $Q$, Violan $C$, Rodriguez-Blanco $T$, Roso-Llorach A, Pons-Vigues $M$, Pujol-Ribera $E$, et al. Multimorbidity patterns in elderly primary health care patients in a south mediterranean european region: a cluster analysis. PLoS One. 2015;10(11)

Doi:

http://dx.doi.org/10.1371/journal.pone.0141155

27. Islas-Granillo H, Eduardo Medina-Solis C, de Lourdes Marquez-Corona $M$, de la Rosa-Santillana $R$, Angel Fernandez-Barrera $M$, Jose Villalobos-Rodelo $J$, et al. Prevalence of multimorbidity in subjects aged $>=60$ years in a developing country. Clin Interv Aging. 2018;13:1129-33. Doi: http://dx.doi.org/10.2147/CIA.S154418

28. Kaplan MS, Huguet N, Newsom JT, McFarland BH. Characteristics of physically inactive older adults with arthritis: results of a population-based study. Prev Med (Baltim). 2003;37(1):61-7. Doi: http://dx.doi.org/10.1016/s0091-7435(03)00059-8

29. Penha JCL, Piçarro IC, Barros Neto TL. Evolução da aptidão física e capacidade funcional de mulheres ativas acima de 50 anos de idade de acordo com a idade cronológica, na cidade de Santos. Cienc Saude Col. 2012;17(1):245-53. Doi: https://doi.org/10.1590/S1413-81232012000100027

30. Barduzzi GO, Rocha Júnior PR, Souza Neto JC, Aveiro MC. Capacidade funcional de idosos com osteoartrite submetidos a fisioterapia aquática e terrestre. Fisioter Mov. 2013;26(2):349-60. Doi: http://dx.doi.org/10.1590/S010351502013000200012

31. Ferreira GD, Silva MC, Rombaldi AJ, Wrege ED, Siqueira FV, Hallal PC. Prevalência de dor nas costas e fatores associados em adultos do Sul do Brasil: estudo de base populacional. Rev Bras Fisioter. 2011;15(1):31-6. Doi: http://dx.doi.org/10.1590/S1413-355520110050 00001

32. Meucci RD, Fassa AG, Faria NM. Prevalence of chronic low back pain: systematic review. Rev Saude Publica. 2015;49:1. Doi: http://dx.doi.org/10.1590/S0034-8910.2015049005874

33. Esquenazi D, Silva SRB, Guimarães MAM. Aspectos fisiopatológicos do envelhecimento humano e quedas em idosos. Rev RUPE. 2014;13(2):11-20. Doi: http://dx.doi.org/10.12957/rhupe.2014.10124

34. Fechine BRA, Trompieri N. O processo de envelhecimento: as principais alterações que acontecem com o idoso com o passar dos anos. Inter Sci Place. 2012;1(20):106-32. Doi http://dx.doi.org/10.6020/1679-9844/2007 
35. Manek NJ, MacGregor AJ. Epidemiology of back disorders: prevalence, risk factors, and prognosis. Curr Opin Rheumatol. 2005;17(2):134-40.

Doi: http://dx.doi.org/10.1097/01.bor.0000154215.08986.06

36. Romero DE, Santana D, Borges P, Marques A, Castanheira D, Rodrigues JM, et al. Sabbadini L. Prevalence, associated factors, and limitations related to chronic back problems in adults and elderly in Brazil. Cad Saude Publica. 2018;34(2):e00012817. Doi: http://dx.doi.org/10.1590/0102-311X00012817

37. Lacourt MX, Marini LL. Decréscimo da função muscular decorrente do envelhecimento e a influência na qualidade de vida do idoso: uma revisão de literatura. RBCEH. 2006;3(1):114-21.

Doi: https://doi.org/10.5335/rbceh.2012.51

38. Alves LC, Leimann BCQ, Vasconcelos MEL, Carvalho MS, Vasconcelos AGG, Fonseca TCO, et al. A influência das doenças crônicas na capacidade funcional dos idosos do Município de São Paulo, Brasil. Cad Sau Pub. 2007;23(8):1924-30. Doi: https://doi.org/10.1590/S0102$\underline{311 \times 2007000800019}$
39. Montez JK, Friedman EM. Educational attainment and adult health: under what conditions is the association causal? Soc Sci Med. 2015;127:1-7. Doi: http://dx.doi.org/10.1016/j.socscimed.2014.12.029

40. Barata RB. Acesso e uso de serviços de saúde: considerações sobre os resultados da Pesquisa de Condições de Vida 2006. São Paulo Perspect. 2008;22(2):19-29.

41. Dias-da-Costa JS, Olinto MTA, Soares SA, Nunes MF, Bagatini $T$, Marues $M C$, et al. Utilização de serviços de saúde pela população adulta de São Leopoldo, Rio Grande do Sul, Brasil: resultados de um estudo transversal. Cad Saude Publica. 2011;27(5):868-76. Doi: https://doi.org/10.1590/S0102$\underline{311 \times 2011000500005}$

42. Pereira MG, Galvão TF. Heterogeneidade e viés de publicação em revisões sistemáticas. Epidemiol Serv Saúde. 2014;23(4):775-8. Doi: https://doi.org/10.5123/S1679$\underline{49742014000400021}$

43. Leopoldino AAO, Diz JBM, Martins VT, Henschke N, Pereira LSM, Dias RC, et al. Prevalência de lombalgia na população idosa brasileira: revisão sistemática com metanálise. Rev Bras Reumatol. 2016;56(3):258-69. Doi: http://dx.doi.org/10.1016/j.rbr.2016.01.004 